\title{
Multilayer-omics analyses of human cancers: exploration of biomarkers and drug targets based on the activities of the International Human Epigenome Consortium
}

\author{
Yae Kanai ${ }^{1,2}{ }^{*}$ and Eri Arai ${ }^{1,2}$ \\ ${ }^{1}$ Division of Molecular Pathology, National Cancer Center Research Institute, Tokyo, Japan \\ ${ }^{2}$ Core Research for Evolutional Science and Technology, Japan Science and Technology Agency, Tokyo, Japan
}

\section{Edited by:}

Yoshimasa Saito, Keio University, Japan

\section{Reviewed by:}

Makoto Chuma, Hokkaido University, Japan

Masaaki Takamura, Niigata University Graduate School of Medical and Dental Sciences, Japan

\section{*Correspondence:}

Yae Kanai, Division of Molecular Pathology, National Cancer Center Research Institute, 5-1-1 Tsukiji, Chuo-ku, Tokyo 104-0045, Japan e-mail:ykanai@ncc.go.jp
Epigenetic alterations consisting mainly of DNA methylation alterations and histone modification alterations are frequently observed in cancers associated with chronic inflammation and/or persistent infection with viruses or other pathogenic microorganisms, or with cigarette smoking. Accumulating evidence suggests that alterations of DNA methylation are involved even in the early and precancerous stages. On the other hand, in patients with cancers, aberrant DNA methylation is frequently associated with tumor aggressiveness and poor patient outcome. Recently, epigenome alterations have been attracting a great deal of attention from researchers who are focusing on not only cancers but also neuronal, immune and metabolic disorders. In order to accurately identify disease-specific epigenome profiles that could be potentially applicable for disease prevention, diagnosis and therapy, strict comparison with standard epigenome profiles of normal tissues is indispensable. However, epigenome mechanisms show heterogeneity among tissues and cell lineages. Therefore, it is not easy to obtain a comprehensive picture of standard epigenome profiles of normal tissues. In 2010, the International Human Epigenome Consortium (IHEC) was established to coordinate the production of reference maps of human epigenomes for key cellular states. In order to gain substantial coverage of the human epigenome, the IHEC has set an ambitious goal to decipher at least 1000 epigenomes within the next 7-10 years. We consider that pathway analysis using genes showing multilayer-omics abnormalities, including genome, epigenome, transcriptome, proteome and metabolome abnormalities, may be useful for elucidating the molecular background of pathogenesis and for exploring possible therapeutic targets for each disease.

Keywords: epigenetics, epigenome, DNA methylation, International Human Epigenome Consortium (IHEC), multilayer/integrated disease omics analyses

\section{MICRO RNAs AND HUMAN DISEASES}

The Encyclopedia of DNA Elements (ENCODE) Consortium ${ }^{1}$ data have revealed in more detail the high degree of complexity of the mammalian transcriptome: $75 \%$ of the genome is transcribed into different types of RNA molecules, e.g., protein-coding, long non-coding, pseudogenes, and small RNA genes (Djebali et al., 2012). RNA molecules show much greater variety than previously suspected. Among such RNA molecules, microRNAs (miRNAs) are non-coding RNAs comprising about 22 nucleotides initially transcribed by RNA polymerase II as primary miRNA (pri-miRNA) molecule precursors that possess a stem loop structure (Jinek and Doudna, 2009). RNase III Drosha acts over

\footnotetext{
Abbreviations: CIMP, CpG island methylator phenotype; COPD, chronic obstructive pulmonary disease; IHEC, International Human Epigenome Consortium; LC, normal lung tissue; $\mathrm{LN}$, non-cancerous lung tissue obtained from patients with lung adenocarcinoma; LT, lung adenocarcinoma tissue; PBAT, post-bisulfite adaptortagging; RC, normal renal cortex tissue; RN, non-cancerous renal cortex tissue obtained from patients with clear cell renal cell carcinoma; RT, clear cell renal cell carcinoma tissue.

${ }^{1}$ https://genome.ucsc.edu/ENCODE/
}

pri-mRNAs generating a pre-miRNA containing the hairpin (Jinek and Doudna, 2009). The pre-miRNAs are then exported to the cytoplasm and processed by Dicer into mature miRNAs, which are subsequently translocated into the RNA-induced silencing complex (RISC; Gomes et al., 2013). Each miRNA has multiple tasks, such as transcriptional repression via binding to partially complementary sequences in the $3^{\prime}$-untranslated regions of the target mRNAs and direct mRNA degradation via binding to perfectly complementary sequences (He and Hannon, 2004). Therefore, deregulation of miRNA levels may disturb the expression profiles in cells, thereby playing a key role in induction of diseases, such as cancers, neurodegenerative diseases, and autoimmune diseases.

\section{EPIGENETICS AND miRNAs}

Saito et al. revealed that treatment with the DNA demethylating agent 5-aza-2'-deoxycytidine and the histone deacetylase inhibitor 4-phenylbutyric acid induced marked changes in the expression profiles of miRNAs in human cancer cell lines. In particular, DNA hypermethylation and induction of active histone marks in the 
promoter region of miR-127 resulted in decreased and increased expression of miR-127, respectively (Saito et al., 2006). Activation of miR-512-5p by epigenetic treatment induced apoptosis of human gastric cancer cell lines via suppression of the MCL1 gene (Saito et al., 2009). In the human colon cancer cell line HCT116, disturbance of miRNA expression patterns has been reported after disruption of both DNA methyltransferase (DNMT) 1 and DNMT3B (Lujambio et al., 2007). Findings accumulated to date clearly indicate that expression levels of multiple miRNAs, such as let-7a-3, miR-1, miR-9-1, miR-9-3, miR-34a, mir34a*, mir34b/c, miR-124a, miR-126. miR127, miR-342, and miR-512-5p, are regulated epigenetically (Saito et al., 2013).

On the other hand, the expression of many proteins involved in epigenetics is regulated by miRNAs. For example, miR-152 acts as a tumor suppressor via suppression of DNMT1 (Huang et al., 2010). The miR-29 family targets DNMT3A and DNMT3B, whereas miR101 targets EZH2 and may alter global chromatin structure (Fabbri et al., 2007). In addition, it has been shown that miRNA has the capacity to recognize chromatin by increasing the methylation of histone, for example through histone $\mathrm{H} 3$ lysine 27 tri-methylation (Kim et al., 2008). Thus the close connection between epigenetic alterations and miRNA dysregulation may have a great impact on human diseases.

\section{PARTICIPATION OF EPIGENETIC ALTERATIONS IN MULTISTAGE HUMAN CARCINOGENESIS}

Epigenetic alterations, consisting mainly of DNA methylation alterations and histone modification alterations, are often observed in cancers that are associated with chronic inflammation and/or persistent infection with viruses, such as hepatitis B or C viruses, Epstein-Barr virus, and human papillomavirus, or with cigarette smoking (Kanai and Hirohashi, 2007). Accumulating evidence suggests that alterations of DNA methylation are involved even in the early and precancerous stages (Arai and Kanai, 2010). On the other hand, in patients with cancers, aberrant DNA methylation is frequently associated with tumor aggressiveness and poor patient outcome (Kanai, 2008). Precancerous conditions showing alterations of DNA methylation may progress rapidly and generate more malignant cancers (Kanai, 2010).

As we described in the webpage of our laboratory ${ }^{2}$, even though genetic alterations, such as activation of oncogenes and inactivation of tumor suppressor genes, have been considered to provide the molecular framework of multistage human carcinogenesis, genetic events alone may not explain the histological heterogeneity underlying the complex biological characteristics of tumors. Therefore, in the 1990s, we began to focus on epigenetic events that can be reversible, in an attempt to explain why cancers show such histopathological heterogeneity. At a time when only two genes, $R B$ and $V H L$, were known as tumor suppressor genes silenced by DNA methylation, we showed for the first time that the $\mathrm{CDH} 1$ gene, which encodes the E-cadherin cell adhesion molecule and acts as tumor suppressor, is silenced by DNA methylation around the promoter region in human cancers (Yoshiura et al., 1995). In 1996, we demonstrated that DNA methylation alterations frequently occurred at multiple loci on chromosome 16, one of the hot spots

\footnotetext{
${ }^{2}$ http://www.ncc.go.jp/en/nccri/divisions/01path/01path01.html
}

for loss of heterozygosity in liver cancers. This preceded loss of heterozygosity even at the chronic hepatitis or liver cirrhosis stages, which are widely considered to be precancerous conditions. This was one of the earliest reports of aberrant DNA methylation at the precancerous stage (Kanai et al., 1996).

Since then, we have reported DNA methylation alterations in tissue specimens at precancerous stages and in cancers using a candidate-gene approach. As an example of inflammationassociated carcinogenesis, ductal adenocarcinomas of the pancreas frequently develop in a background of chronic pancreatitis. Under these conditions, at least a proportion of peripheral pancreatic duct epithelia may be at the precancerous stage. It has been reported that the average number of methylated tumor-related genes, the incidence of DNA methylation of at least one of such genes, and the expression level of DNMT1 protein are increased in pancreatic duct epithelia with an inflammatory background, and in another precancerous lesion, pancreatic intraductal neoplasia (PanIN), in comparison with normal pancreatic duct epithelia (Peng et al., 2006).

Urothelial carcinomas of the urinary bladder, renal pelvis, and ureter are clinically remarkable because of their multicentricity and tendency to recur. Such multiplicity may be attributable to the "field effect." Even non-cancerous urothelia showing no marked histological findings from patients with urothelial carcinomas can be considered precancerous, because they may have been exposed to carcinogens in the urine. It has been reported that the average number of methylated tumor-related genes and the expression level of DNMT1 protein are increased in non-cancerous urothelia showing no marked histological findings from patients with urothelial carcinomas, in comparison with normal urothelia from patients without urothelial carcinomas (Nakagawa et al., 2005). Thus, overexpression of the major DNMT, DNMT1, may result in accumulated hypermethylation of DNA for tumor-related genes (Etoh et al., 2004). On the other hand, splicing alteration of DNMT3B may induce chromosomal instability through DNA hypomethylation of pericentromeric satellite regions (Saito et al., 2002).

As we described in the webpage of our laboratory ${ }^{2}$, after genome-wide epigenetic (epigenome) analysis had become practical, we employed the bacterial artificial chromosome array-based methylated $\mathrm{CpG}$ island amplification (BAMCA) method for overviewing the DNA methylation tendency of large individual chromosomal regions. Although precancerous conditions in the kidney have rarely been described, despite the lack of any marked histological findings or association with chronic inflammation or persistent infection with pathogens, it can be considered that noncancerous renal cortex tissue obtained from patients with renal cancers is already at the precancerous stage showing genome-wide DNA methylation alterations (Arai et al., 2006). We showed that DNA methylation profiles at the precancerous stage are inherited by renal cancers developing in individual patients, and that DNA methylation alterations at the precancerous stage determine both the aggressiveness of subsequently developing cancers and patient outcome through inducing further epigenetic and genetic alterations (Arai et al., 2009a). In addition, we have developed indicators for carcinogenetic risk estimation in patients with chronic hepatitis and liver cirrhosis (Arai et al., 2009b), indicators 
for estimating the risk of development of urothelial carcinomas that can be determined from urine samples (Nishiyama et al., 2010), diagnostic markers of pancreatic cancer that can be assessed from specimens of pancreatic juice (Gotoh et al., 2011), and indicators for prognostication of kidney, liver, pancreas, and urinary bladder cancers based on DNA methylation profiling. Based on these findings, we have filed patent applications for epigenome diagnosis techniques, and are now attempting to apply them practically.

\section{ACTIVITIES OF THE INTERNATIONAL HUMAN EPIGENOME CONSORTIUM (IHEC)}

Recently, epigenome alterations have been attracting a great deal of attention from researchers who are focusing on not only cancers but also neuronal, immune, and metabolic disorders. On the basis of epigenome profiling, attempts are now being made to elucidate the molecular pathogenesis of such diseases and to explore possible biomarkers and drug targets. In order to accurately identify such disease-specific epigenome profiles that could be potentially applicable for disease prevention, diagnosis, and therapy, strict comparison with standard epigenome profiles of normal tissues is indispensable. However, epigenome mechanisms show heterogeneity among tissues and cell lineages. Therefore, it is not easy to obtain a comprehensive picture of standard epigenome profiles of normal tissues. Based on improvements in next-generation sequencing technology, international collaboration will likely help to reveal standard epigenome profiles.

In 2010, the IHEC was established by researchers and founding agencies from Canada, South Korea, the EU, Italy, Germany, Japan, and the USA (Bae, 2013). As described in the webpage of IHEC ${ }^{3}$, the primary goal of the IHEC is "to coordinate the production of reference maps of human epigenomes for key cellular states that are relevant to health and diseases." In order to achieve substantial coverage of the human epigenome, the IHEC has set an ambitious goal to decipher at least 1000 epigenomes ${ }^{3}$. To attain this goal, IHEC will use robust techniques to generate (1) high-resolution maps of histone modifications, H3K4me3, H3K9me3, H3K27me3, H3K27ac, H3K4me1, and H3K36me3, (2) high-resolution DNA methylation maps, (3) landmark maps of transcription start sites for all protein-encoding genes, and (4) a comprehensive catalog of non-coding and small RNAs and their patterns of expression ${ }^{3}$. The target cell types being studied by each team in the participating countries are shown on the IHEC website ${ }^{4}$.

In Japan, three Japanese IHEC teams ${ }^{5}$ including our team are supported by the Core Research for Evolutional Science and Technology division of the Japan Science and Technology Agency. To strengthen the research bases for cancers of digestive organs, including hepatocellular carcinomas and gastric carcinomas, which show high incidences in Japan, we are now performing standard epigenome analyses of normal epithelial cell lineages in digestive organs (Figure 1). Target cells of sufficient quality and quantity are being obtained from materials surgically resected from a range of Japanese patients. For example, for liver, we have

\footnotetext{
${ }^{3}$ http://ihec-epigenomes.org/about/objectives/

${ }^{4}$ http://ihec-epigenomes.org/research/cell-types/

${ }^{5}$ http://crest-ihec.jp/
}

obtained samples of normal liver tissue distant from sites of liver metastases from primary colon cancers in partial hepatectomy specimens from patients without viral hepatitis, chronic hepatitis, or liver cirrhosis. To isolate hepatocytes, we have performed collagenase perfusion of cannulated branches of the hepatic vein, followed by low-velocity centrifugation. On average, more than $10^{7}$ dispersed cells can be obtained from each case, and immunocytochemistry has confirmed that the hepatocytes are more than 95\% pure. In the stomach and colorectum, we initially employ the crypt isolation technique and collagenase digestion. Thereafter, each normal cell lineage is purified by fluorescence activated cell sorting using appropriate antibodies.

Members of our IHEC team have originally developed the postbisulfite adaptor-tagging method (PBAT), which is an efficient library preparation method for whole-genome bisulfite sequencing (Miura et al., 2012). For the PBAT method, we first perform bisulfite modification followed by adaptor ligation using random priming. The PBAT method minimally requires sub-microgram DNA for mammalian whole-genome bisulfite sequencing without global PCR amplification. A good correlation of the DNA methylation pattern was observed among PBAT, the standard Methyl C-seq methodology developed by Lister et al. (2008), and the Illumina beads chip Infinium assay. The PBAT method is advantageous in that it requires only a small amount of genomic DNA but has good coverage of GC-rich regions, especially in $\mathrm{CpG}$ islands and generich chromosomes. We now propose to make the PBAT method one of the standard protocols for IHEC. Under the supervision of the IHEC, we intend to disclose the data we obtain through the National Bioscience Database Center supported by the Japan Science and Technology Agency. Accurate standard epigenome profiles of digestive organ epithelial cells obtained through IHEC activities will be used to explore more useful biomarkers and drug targets of digestive organ cancers.

\section{MULTILAYER/INTEGRATIVE DISEASE OMICS ANALYSES FOR EXPLORATION OF BIOMARKERS AND DRUG TARGETS}

Recently big data analysis has impacted various fields of bioscience, especially disease research. It may not be appropriate to perform epigenome analysis including miRNA analysis using clinical samples. Instead, simultaneous multilayer/integrative disease omics analyses would seem more appropriate, including genome, epigenome, transcriptome, proteome, and metabolome analyses for exploration of drug targets. Since 2010, researchers at six National Centers in Japan, i.e., the National Cancer Center, National Cerebral and Cardiovascular Center, National Center for Neurology and Psychiatry, National Center for Global Health and Medicine, National Center for Child Health and Development and National Center for Geriatrics and Gerontology, have been engaged in a research project "Comprehensive exploration of drug targets based on multilayer/integrative disease omics analyses" supported by the Program for Promotion of Fundamental Studies in Health Sciences of the National Institute of Biomedical Innovation (NiBio) (Figure 2). This project has been divided among a number of centers specializing in genome, epigenome, transcriptome, proteome, and metabolome analyses of tissue specimens from patients with various diseases that show a high incidence in the Japanese population. Tissue and body fluid 

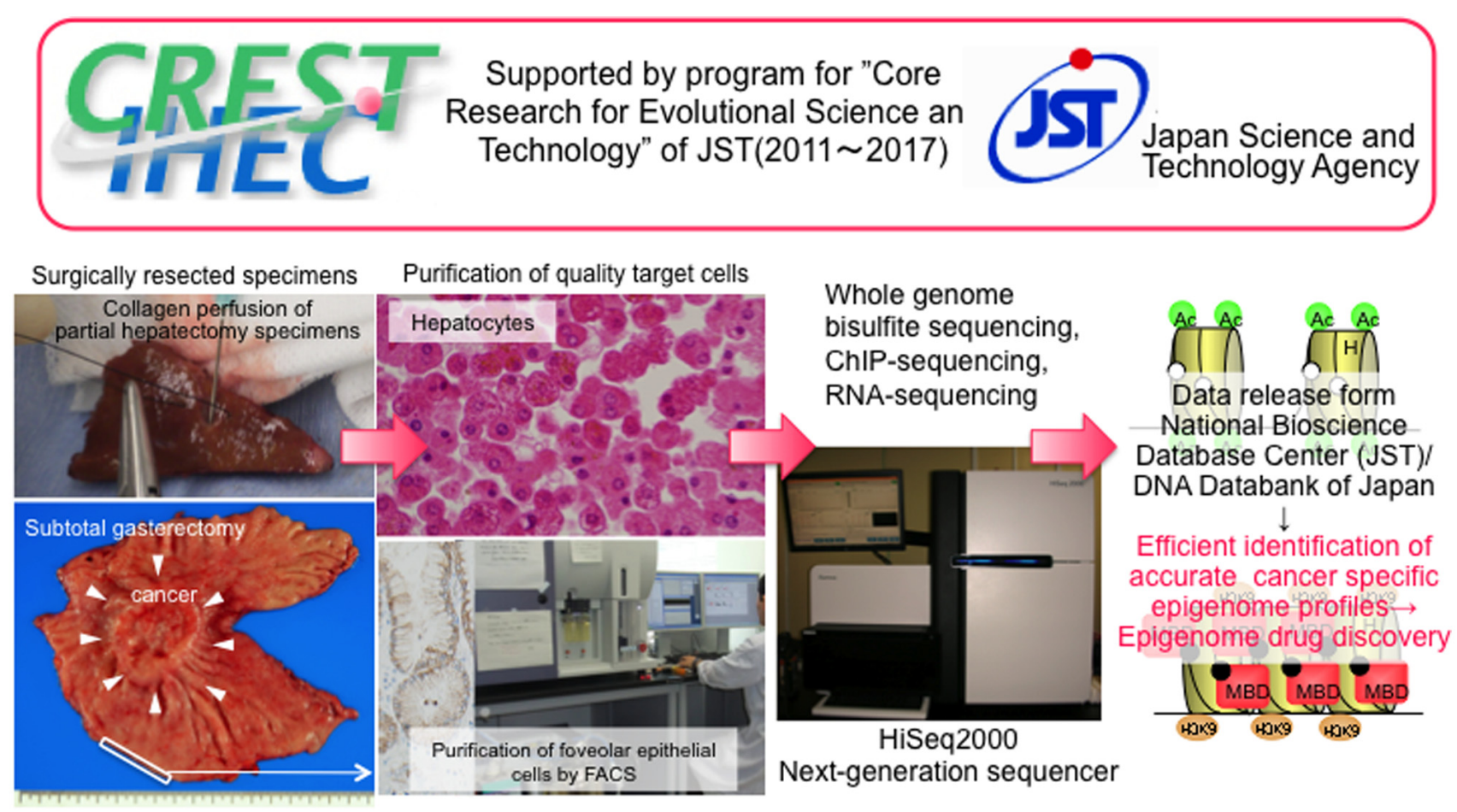

FIGURE 1 | Activity of our team, one of the Japan Teams of the International Human Epigenome Consortium supported by the Core Research for Evolutional Science and Technology division of the Japan Science and Technology Agency (modified from the webpage of our laboratory [http://www.ncc.go.jp/en/nccri/divisions/01path/01path01.html]). We are now performing whole-genome bisulfite sequencing using the post-bisulfite adaptor-tagging method, chromatin immunoprecipitation-sequencing, and RNA-sequencing of purified target cells, i.e., hepatocytes and other live cell lineages, foveolar epithelial cells, and other gastric epithelial cell lineages, and absorptive epithelial cells from the ascending and descending colon and rectum. Accurate epigenome profiling of normal cells will allow the identification of disease-specific epigenome profiles, thus facilitating a potential breakthrough in the prevention, diagnosis, and therapy of diseases. specimens, cultured cells and animal models of adult cancers, infant leukemia, allergic disease, dilated cardiomyopathy, aortic aneurysm, epilepsy, obesity, non-alcoholic steatohepatitis, spinal canal stenosis, and Alzheimer's disease have been subjected to multilayer-omics analyses. As we described in the webpage of our laboratory ${ }^{2}$, we are especially focusing on molecules or molecular pathways which are impaired as a result of multiple mechanisms, such as events in all five omics layers, which may participate in the molecular pathogeneses of diseases and might become potential biomarkers and/or druggable targets (Figure 2).

With regard to epigenome analysis of adult cancers in this research project, 414 lung tissue specimens including normal lung tissue (LC) obtained from patients without any primary lung tumor, non-cancerous lung tissue (LN) obtained from patients with lung adenocarcinomas, and lung adenocarcinoma tissue (LT) itself have been subjected to single-CpG resolution Infinium assay. DNA methylation alterations on many probes were evident in LN samples relative to LC samples, and were inherited by, or strengthened in, LT samples. Unsupervised hierarchical clustering using DNA methylation levels in LN samples subclustered patients into clusters I, II, and III. Lung adenocarcinomas in cluster I developed from an inflammatory background in chronic obstructive pulmonary disease (COPD) in heavy smokers, and were locally invasive. Most patients in cluster II were non-smokers and had a favorable outcome. Lung adenocarcinomas in cluster III were most aggressive cancers in light smokers that developed before accumulation of the long-term effects of cigarette smoking, and were probably due to the direct actions of carcinogens, rather than the effects of inflammation. DNA methylation profiles reflecting carcinogenetic factors such as smoking and COPD appear to be established in LNs and may determine the aggressiveness of tumors developing in individual patients, and thus patient outcome (Sato et al., 2014). Among the genes for which DNA methylation status in LN samples was significantly correlated with recurrence of lung adenocarcinomas in individual patients, we focused on $A D C Y 5, E V X 1$, and other genes that were involved in apoptosis and cell adhesion. The mRNA expression levels of these genes were directly regulated by DNA methylation, and a decrease of their mRNA expression in LT samples was significantly correlated with tumor aggressiveness (Sato et al., 2013). When these genes were ectopically expressed in lung cancer cell lines, growth suppression, and apoptosis were induced, indicating that these genes could become therapeutic targets of lung adenocarcinomas.

With regard to epigenome analysis during renal carcinogenesis, 245 renal tissue specimens including normal renal cortex tissue (RC) obtained from patients without any primary renal cancer, non-cancerous renal cortex tissue (RN) obtained from patients with clear cell renal cell carcinomas, and clear cell 


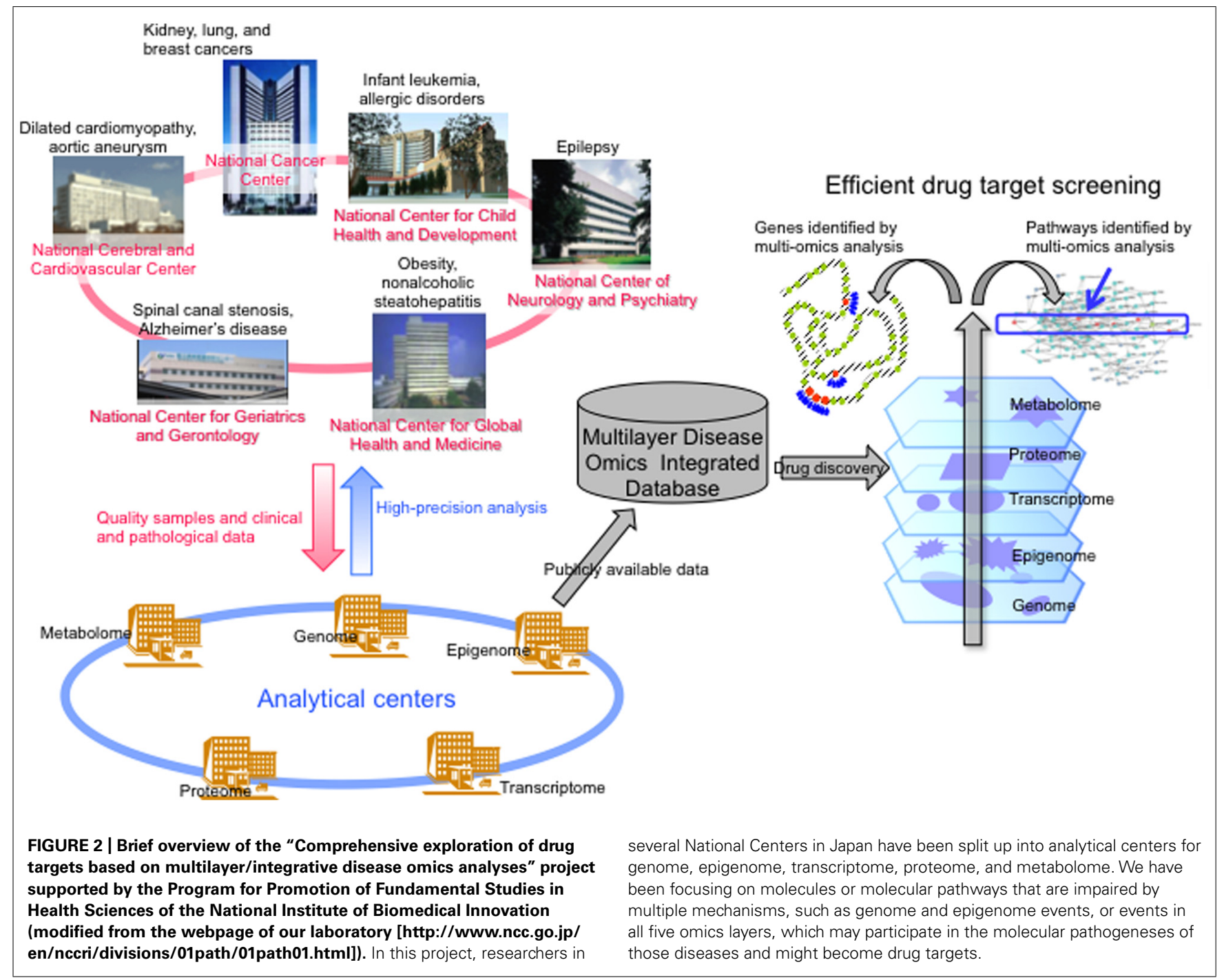

renal cell carcinoma tissue (RT) itself were subjected to the Infinium assay. DNA methylation levels at multiple Infinium probe sites were already altered in $\mathrm{RN}$ samples relative to RC samples. Unsupervised hierarchical clustering analysis based on DNA methylation levels at the CpG sites where DNA methylation alterations had occurred even in $\mathrm{RN}$ samples and were inherited by, and strengthened in, RT samples divided the clear cell renal cell carcinomas into $\mathrm{CpG}$ island methylator phenotype (CIMP)-positive and -negative clusters (Arai et al., 2012). Clinicopathologically aggressive cancers were accumulated in the CIMP-positive cluster, where the cancer-free and overall survival rates of the patients were significantly lower than in the CIMPnegative cluster. FAM150A, GRM6, ZNF540, ZFP42, ZNF154, RIMS4, PCDHAC1, KHDRBS2, ASCL2, KCNQ1, PRAC, WNT3A, TRH, FAM78A, ZNF671, SLC13A5, and NKX6-2 have been identified as renal cell carcinoma-specific CIMP marker genes (Arai et al., 2012). Since CIMP-positive renal cell carcinomas show tumor aggressiveness and poorer patient outcome, we established criteria for prognostication of patients with clear cell renal cell carcinomas using renal cell carcinoma-specific CIMP marker genes. We are now performing pathway analysis based on a Bayesian estimation model using multiple genes showing frequent mutations and alterations of expression at the mRNA, miRNA, and protein levels based on multilayer-omics analyses in each of the CIMPnegative and CIMP-positive renal cell carcinomas for exploration of possible drug targets.

\section{PERSPECTIVES}

Once DNA methylation alterations occur during multistage carcinogenesis, such alterations are stably preserved on DNA double strands through maintenance methylation mechanisms by DNMT1. Therefore, stable stratification of cancers reflecting clinicopathological diversity may be possible based on epigenome profiling. Genes showing epigenome alterations, such as CIMP-marker genes, may become excellent biomarkers discriminating each tumor type stratified on the basis of epigenome profiling. We consider that pathway analysis using genes showing multilayer-omics abnormalities after stratification based on epigenome profiling may be useful for elucidating the molecular background of carcinogenetic pathways 
and for exploring possible therapeutic targets for each tumor type.

\section{ACKNOWLEDGMENTS}

This study was supported by the Core Research for Evolutional Science and Technology (CREST) division "Development of Fundamental Technologies for Diagnosis and Therapy Based upon Epigenome Analysis" of the Japan Science and Technology Agency (JST), the Program for Promotion of Fundamental Studies in Health Sciences from the NiBio, a Grant in Aid for the Third Term Comprehensive 10-Year Strategy for Cancer Control from the Ministry of Health, Labor and Welfare of Japan, and Grants in Aid for Scientific Research (B) and (C) from the Japan Society for the Promotion of Science (JSPS). Tissue specimens were provided by the National Cancer Center Biobank, which is supported by the National Cancer Center Research and Development Fund, Japan.

\section{REFERENCES}

Arai, E., Chiku, S., Mori, T., Gotoh, M., Nakagawa, T., Fujimoto, H., et al. (2012). Single-CpG-resolution methylome analysis identifies clinicopathologically aggressive $\mathrm{CpG}$ island methylator phenotype clear cell renal cell carcinomas. Carcinogenesis 33, 1487-1493. doi: 10.1093/carcin/bgs177

Arai, E., and Kanai, Y. (2010). DNA methylation profiles in precancerous tissue and cancers: carcinogenetic risk estimation and prognostication based on DNA methylation status. Epigenomics 2, 467-481. doi: 10.2217/epi.10.16

Arai, E., Kanai, Y., Ushijima, S., Fujimoto, H., Mukai, K., and Hirohashi, S. (2006). Regional DNA hypermethylation and DNA methyltransferase (DNMT) 1 protein overexpression in both renal tumors and corresponding nontumorous renal tissues. Int. J. Cancer 119, 288-296. doi:10.1002/ijc.21807

Arai, E., Ushijima, S., Fujimoto, H., Hosoda, F., Shibata, T., Kondo, T., et al. (2009a). Genome-wide DNA methylation profiles in both precancerous conditions and clear cell renal cell carcinomas are correlated with malignant potential and patient outcome. Carcinogenesis 30, 214-221. doi: 10.1093/carcin/bgn268

Arai, E., Ushijima, S., Gotoh, M., Ojima, H., Kosuge, T., Hosoda, F., et al. (2009b). Genome-wide DNA methylation profiles in liver tissue at the precancerous stage and in hepatocellular carcinoma. Int. J. Cancer 125, 2854-2862. doi: $10.1002 /$ ijc. 24708

Bae, J. B. (2013). Perspectives of international human epigenome consortium. Genomics Inform. 11, 7-14. doi: 10.5808/GI.2013.11.1.7

Djebali, S., Davis, C. A., Merkel, A., Dobin, A., Lassmann, T., Mortazavi, A., et al. (2012). Landscape of transcription in human cells. Nature 489, 101-108. doi: 10.1038 /nature 11233

Etoh, T., Kanai, Y., Ushijima, S., Nakagawa, T., Nakanishi, Y., Sasako, M., et al. (2004). Increased DNA methyltransferase 1 (DNMT1) protein expression correlates significantly with poorer tumor differentiation and frequent DNA hypermethylation of multiple CpG islands in gastric cancers. Am. J. Pathol. 164, 689-699. doi: 10.1016/S0002-9440(10)63156-2

Fabbri, M., Garzon, R., Cimmino, A., Liu, Z., Zanesi, N., Callegari, E., et al. (2007). MicroRNA-29 family reverts aberrant methylation in lung cancer by targeting DNA methyltransferases 3A and 3B. Proc. Natl. Acad. Sci. U.S.A. 104, 1580515810. doi:10.1073/pnas.0707628104

Gomes, A. Q., Nolasco, S., and Soares, H. (2013). Non-coding RNAs: multitasking molecules in the cell. Int. J. Mol. Sci. 14, 16010-16039. doi: 10.3390/ijms140816010

Gotoh, M., Arai, E., Wakai-Ushijima, S., Hiraoka, N., Kosuge, T., Hosoda, F., et al. (2011). Diagnosis and prognostication of ductal adenocarcinomas of the pancreas based on genome-wide DNA methylation profiling by bacterial artificial chromosome array-based methylated CpG island amplification. J. Biomed. Biotechnol. 2011, 780836. doi: 10.1155/2011/780836

He, L., and Hannon, G. J. (2004). MicroRNAs: small RNAs with a big role in gene regulation. Nat. Rev. Genet. 5, 522-531. doi: 10.1038/nrg1379

Huang, J., Wang, Y., Guo, Y., and Sun, S. (2010). Down-regulated microRNA-152 induces aberrant DNA methylation in hepatitis B virus-related hepatocellular carcinoma by targeting DNA methyltransferase 1. Hepatology 52, 60-70. doi: 10.1002/hep.23660
Jinek, M., and Doudna, J. A. (2009). A three-dimensional view of the molecular machinery of RNA interference. Nature 457, 405-412. doi: 10.1038/ nature 07755

Kanai, Y. (2008). Alterations of DNA methylation and clinicopathological diversity of human cancers. Pathol. Int. 58, 544-558. doi: 10.1111/j.14401827.2008.02270.x

Kanai, Y. (2010). Genome-wide DNA methylation profiles in precancerous conditions and cancers. Cancer Sci. 101, 36-45. doi: 10.1111/j.1349-7006.2009. 01383.x

Kanai, Y., and Hirohashi, S. (2007). Alterations of DNA methylation associated with abnormalities of DNA methyltransferases in human cancers during transition from a precancerous to a malignant state. Carcinogenesis 28, 2434-2442. doi: 10.1093/carcin/bgm206

Kanai, Y., Ushijima, S., Tsuda, H., Sakamoto, M., Sugimura, T., and Hirohashi, S. (1996). Aberrant DNA methylation on chromosome 16 is an early event in hepatocarcinogenesis. Jpn. J. Cancer Res. 87, 1210-1217. doi: 10.1111/j.13497006.1996.tb03135.x

Kim, D. H., Saetrom, P., Snøve, O. Jr., and Rossi, J. J. (2008). MicroRNA-directed transcriptional gene silencing in mammalian cells. Proc. Natl. Acad. Sci. U.S.A. 105, 16230-16235. doi: 10.1073/pnas.0808830105

Lister, R., O’Malley, R. C., Tonti-Filippini, J., Gregory, B. D., Berry, C. C., Millar, A. H., et al. (2008). Highly integrated single-base resolution maps of the epigenome in Arabidopsis. Cell 133, 523-536. doi: 10.1016/j.cell.2008.03.029

Lujambio, A., Ropero, S., Ballestar, E., Fraga, M. F., Cerrato, C., Setién, F., et al. (2007). Genetic unmasking of an epigenetically silenced microRNA in human cancer cells. Cancer Res. 67, 1424-1429. doi: 10.1158/0008-5472.CAN06-4218

Miura, F., Enomoto, Y., Dairiki, R., and Ito, T. (2012). Amplification-free wholegenome bisulfite sequencing by post-bisulfite adaptor tagging. Nucleic Acids Res. 40, e136. doi: 10.1093/nar/gks454

Nakagawa, T., Kanai, Y., Ushijima, S., Kitamura, T., Kakizoe, T., and Hirohashi, S. (2005). DNA hypermethylation on multiple CpG islands associated with increased DNA methyltransferase DNMT1 protein expression during multistage urothelial carcinogenesis. J. Urol. 173, 1767-1771. doi: 10.1097/01.ju.0000154632.11824.4d

Nishiyama, N., Arai, E., Chihara, Y., Fujimoto, H., Hosoda, F., Shibata, T., et al. (2010). Genome-wide DNA methylation profiles in urothelial carcinomas and urothelia at the precancerous stage. Cancer Sci. 101, 231-240. doi: 10.1111/j.13497006.2009.01330.x

Peng, D. F., Kanai, Y., Sawada, M., Ushijima, S., Hiraoka, N., Kitazawa, S., etal. (2006). DNA methylation of multiple tumor-related genes in association with overexpression of DNA methyltransferase 1 (DNMT1) during multistage carcinogenesis of the pancreas. Carcinogenesis 27, 1160-1168. doi: 10.1093/carcin/bgi361

Saito, Y., Kanai, Y., Sakamoto, M., Saito, H., Ishii, H., and Hirohashi, S. (2002). Overexpression of a splice variant of DNA methyltransferase 3b, DNMT3b4, associated with DNA hypomethylation on pericentromeric satellite regions during human hepatocarcinogenesis. Proc. Natl. Acad. Sci. U.S.A. 99, 10060-10065. doi: 10.1073/pnas.152121799

Saito, Y., Liang, G., Egger, G., Friedman, J. M., Chuang, J. C., and Coetzee. G. A., et al. (2006). Specific activation of microRNA-127 with downregulation of the proto-oncogene BCL6 by chromatin-modifying drugs in human cancer cells. Cancer Cell 9, 435-443. doi: 10.1016/j.ccr.2006.04.020

Saito, Y., Saito, H., Liang, G., and Friedman, J. M. (2013). Epigenetic alterations and microRNA misexpression in cancer and autoimmune diseases: a critical review. Clin. Rev. Allergy Immunol. doi: 10.1007/s12016-013-8401-z [Epub ahead of print].

Saito, Y., Suzuki, H., Tsugawa, H., Nakagawa, I., Matsuzaki, J., Kanai, Y., et al. (2009). Chromatin remodeling at Alu repeats by epigenetic treatment activates silenced microRNA-512-5p with downregulation of Mcl-1 in human gastric cancer cells. Oncogene 28, 2738-2744. doi: 10.1038/onc.2009.140

Sato, T., Arai, E., Kohno T., Takahashi, Y., Miyata, S., Tsuta, K., et al. (2014). Epigenetic clustering of lung adenocarcinomas based on DNA methylation profiles in adjacent lung tissue: its correlation with smoking history and chronic obstructive pulmonary disease. Int. J. Cancer doi: 10.1002/ijc.28684

Sato, T., Arai, E., Kohno, T., Tsuta, K., Watanabe, S., Soejima, K., et al. (2013). DNA methylation profiles at precancerous stages associated with recurrence of lung adenocarcinoma. PLoS ONE 8:e59444. doi: 10.1371/journal.pone.0059444 
Yoshiura, K., Kanai, Y., Ochiai, A., Shimoyama, Y., Sugimura, T., and Hirohashi, S. (1995). Silencing of the E-cadherin invasion-suppressor gene by CpG methylation in human carcinomas. Proc. Natl. Acad. Sci. U.S.A. 92, 7416-7419. doi: 10.1073/pnas.92.16.7416

Conflict of Interest Statement: The authors declare that the research was conducted in the absence of any commercial or financial relationships that could be construed as a potential conflict of interest.

Received: 14 January 2014; accepted: 24 January 2014; published online: 14 February 2014.
Citation: Kanai Y and Arai E (2014) Multilayer-omics analyses of human cancers: exploration of biomarkers and drug targets based on the activities of the International Human Epigenome Consortium. Front. Genet. 5:24. doi: 10.3389/fgene.2014.00024 This article was submitted to Epigenomics and Epigenetics, a section of the journal Frontiers in Genetics.

Copyright (C) 2014 Kanai and Arai. This is an open-access article distributed under the terms of the Creative Commons Attribution License (CC BY). The use, distribution or reproduction in other forums is permitted, provided the original author(s) or licensor are credited and that the original publication in this journal is cited, in accordance with accepted academic practice. No use, distribution or reproduction is permitted which does not comply with these terms. 